\title{
Salivary BDNF and Cortisol Responses During High-Intensity Exercise and Official Basketball Matches in Sedentary Individuals and Elite Players
}

\author{
by \\ Alexandre Moreira ${ }^{1}$, Marcelo Saldanha Aoki ${ }^{2}$, Ademir Felipe Schultz de Arruda ${ }^{1}$, \\ Daniel Gomes da Silva Machado3, Hassan Mohamed Elsangedy3, \\ Alexandre Hideki Okano 3,4
}

\begin{abstract}
Salivary cortisol increases in response to stressors, including physical exertion and psychological stress associated with sports competition. In addition, stress may induce change in brain-derived neurotrophic factor (BDNF). However, there are still no data available to compare the salivary BDNF level in sedentary male individuals and elite team-sport male athletes, regularly involved in activities that require elevated attention and concentration. This information could contribute to the advance of understanding of the effect of regular exercise on the salivary level of $B D N F$, the pre-to-post change in salivary BDNF during exercise, and the association between salivary cortisol and salivary BDNF responses to physical exercise. Therefore, this study aimed to compare the concentration of salivary cortisol and BDNF, before and after exercise, in sedentary individuals and elite male basketball players. The sedentary group (23.0 \pm 4.2 yrs) performed a high-intensity exercise protocol and the basketball players (18.6 \pm 0.5 yrs) participated in three official basketball matches. Saliva samples were tested for cortisol and BDNF using ELISA. A significant increase in salivary cortisol from pre- to post-match was observed only for the basketball players $(p<0.05)$. Basketball players also presented a higher salivary BDNF concentration for both resting (pre) and post-physical exercise $(p<0.05)$; however, no change in pre- to post-exercise salivary BDNF was observed for either group $(p>0.05)$. Elevated BDNF in athletes may be associated to their repeated exposure to stressful competition situations. The current findings also suggest that different mechanisms might be involved in salivary cortisol and BDNF responses during physical exercise.
\end{abstract}

Key words: saliva, team-sports, stress, athletes, neuroendocrinology, exercise.

\section{Introduction}

The hypothalamic-pituitary-adrenal (HPA) axis, in conjunction with the sympathetic system, connects the brain with the periphery of the body. HPA activity can be assessed by means of salivary cortisol (Törnhage, 2009) and previous studies have shown that salivary cortisol is a better measure of adrenal cortical function than serum cortisol (Aardal and Holm, 1995); it has also been suggested that salivary cortisol provides a more sensitive marker of changes in cortisol than its serum equivalent (Gozansky et al., 2005). Salivary cortisol increases in response to stressors, including physical exertion (Papacosta and Nassis, 2011). Exercise, per se, may represent a potent physiological stressor (Gozansky et al., 2005) that may increase cortisol concentration,

\footnotetext{
1 - Department of Sport, School of Physical Education and Sport, University of Sao Paulo, Sao Paulo, Brazil.

2 - School of Arts, Sciences and Humanities, University of Sao Paulo, Sao Paulo, Brazil.

3 - Federal University of Rio Grande do Norte, Dept. of Physical Education, Natal-RN, Brazil.

4 - Federal University of ABC, Sao Paulo, Brazil.
} 
cardiovascular activity, the glucose level and antiinflammatory responses (Sapolsky et al., 2000). In addition, results from studies performed in a sport context suggest that a competition environment can induce a greater increase in salivary cortisol that is likely associated with the additional psychological stress generated in response to official competitions. For example, a marked increment in salivary cortisol concentration has been verified prior to a real weightlifting event versus a simulated event (Crewther et al., 2011), during official Jiu-Jitsu matches as opposed to simulated training matches (Moreira et al., 2012c), and for official basketball matches compared to simulated matches (Moreira et al., 2012a; Moreira et al., 2012b).

There is also mounting evidence to indicate that physical stress (e.g. physical exercise) may induce changes in brain-derived neurotrophic factor (BDNF) in humans (Tirassa et al., 2012) and rodents (Russo-Neustadt et al., 2001). BDNF is the most abundant neurotrophin in the central nervous system (Hofer et al., 1990), and is involved in survival, maintenance, and neural transmission in the neural cell (Lewin and Barde, 1996). BDNF has been suggested to be the neurotrophin that is most sensitive to exercise (Vaynman et al., 2006). Exercise-induced changes in BDNF have been examined using different exercise modes and intensities. For instance, studies focusing on the effects of acute bouts of aerobic exercise have demonstrated transient and moderate increases in the plasma BDNF level in healthy males (Rojas-Vega et al., 2006; Tang et al., 2008). Ferris et al. (2007) showed that the intensity of the exercise played a key role in eliciting an acute change in the BDNF level. Nevertheless, the effect of physical activity on the BDNF level is still controversial. For example, Nofuji et al. (2008) demonstrated that serum BDNF was lower in trained men compared to sedentary individuals. Contrary to these findings, Oztasyonar (2016) found that athletes had a higher basal blood BDNF level than sedentary individuals, and speculated that this result might be explained by the protective role of BDNF against brain damage or the maintenance of higher attention or concentration, as elevated BDNF concentration was found in combat sport athletes.

Besides the presence of BDNF in serum and plasma, BDNF has been detected in human saliva by Mandel et al. (2009) through immunoblotting and enzyme digestions. Tirassa et al. (2012), using a commercial ELISA kit, confirmed the findings of Mandel et al. (2009) in healthy young women, and showed that salivary $\mathrm{BDNF}$ release is influenced by the circadian rhythm, with a decline over the course of the day. The authors reported a mean value of approximately $400 \mathrm{pg} / \mathrm{ml}$ and close to $200 \mathrm{pg} / \mathrm{ml}$ at 9:00 am and 8:00 pm, respectively. Data from other studies using commercial ELISA kits showed that BDNF concentration varied substantially among the investigations. For instance, Saruta et al. (2012) reported values of $52.64 \pm 8.42 \mathrm{pg} / \mathrm{ml}$ and $40.76 \pm 4.83 \mathrm{pg} / \mathrm{ml}$ in females and males, respectively. A lower value was reported by Matsuki et al. (2014) who showed a mean salivary BDNF concentration ranging from $14.91 \pm 7.70 \mathrm{pg} / \mathrm{ml}$ to $22.30 \pm 29.99 \mathrm{pg} / \mathrm{ml}$ on the 13th-16th days and 21st -24th days, respectively, of a normal menstrual cycle in healthy women. While this diversity in reported salivary BDNF concentration suggests that salivary BDNF may be measurable in human using commercial kits, these findings also suggest that caution is needed when comparing different studies that have adopted different procedures for the collection, storage, and processing of BDNF in saliva samples.

Despite the above-mentioned findings for salivary BDNF in healthy adults, there are still no data available to compare the salivary BDNF level in sedentary male individuals and elite team-sport male athletes regularly involved in activities that require elevated attention and concentration, in conjunction with the high psychophysiological demands inherent to official competitions. This information could contribute to the advance of understanding of the effect of regular physical activity on the salivary level of BDNF, the change in salivary BDNF during exercise, including a real sports environment, and the association between salivary cortisol and salivary BDNF responses to physical exercise.

Therefore, the aim of this study was to compare the concentration of salivary cortisol and BDNF before and after physical exercise in sedentary individuals and elite male basketball players, and to examine the relationship between salivary cortisol and BDNF responses. It was hypothesized that athletes would present a higher 
resting salivary BDNF concentration, compared to sedentary individuals, and that salivary cortisol and salivary BDNF would increase from pre- to post physical exercise.

\section{Methods}

\section{Participants}

Thirteen young adult male sedentary volunteers $(23.0 \pm 4.2 \mathrm{yrs})$ and 20 elite male basketball players from the same team (18.6 \pm 0.5 yrs; body height, $192 \pm 7 \mathrm{~cm}$; body mass, $88.9 \pm$ $14.5 \mathrm{~kg}$ ) voluntarily agreed to participate in the study. The control group was classified as sedentary according to the World Health Organization (1995). The basketball players were members of the same team, which was ranked $1^{\text {st }}$ in the under-19 State Basketball Championship during the period investigated. Each player maintained their normal training schedule in the weeks leading up to the investigated match, which remained relatively stable in terms of training mode, frequency and volume across the entire competition season. Habitually, on a weekly basis, the assessed players participated in 1 or 2 training sessions per day ( 90 min per session), 5 days per week and one official match. Training sessions usually consisted of basketball drills, tactics, sprints, intermittent running exercises and specific conditioning drills, as well as weight training and plyometrics. The two additional inclusion criteria for the present investigation, besides being part of the assessed basketball team were: 1) participation in the 3 assessed official matches; 2) provision of saliva samples at all required time-points. Data from 12 basketball players were retained for analysis, according to these criteria (data from 8 players were excluded from the analysis due to limited participation in the 3 matches assessed). All the players were familiarized with the procedures. Data from 12 sedentary individuals were retained for analysis. Data from 1 participant was excluded due to a high intra-assay coefficient of variation for cortisol concentration (>2SD). After being made aware of the study purpose and procedures, the subjects signed a written informed consent form, approved by the Institutional Research Ethics Committee.

\section{Procedures}

\section{Sedentary group}

Participants in the sedentary group visited the lab on 4 occasions. On the $1^{\text {st }}$ visit, they were informed about the study's purpose and procedures, and signed their written informed consent. On the $2^{\text {nd }}$ visit, volunteers performed a maximal incremental exercise in order to determine the heart rate variability threshold $\left(\mathrm{HRV}_{\mathrm{TH}}\right)$ using a digital heart rate (HR) monitor. On the $3^{\text {rd }}$ visit, volunteers performed an exercise protocol at a constant load corresponding to $120 \%$ of $\mathrm{HRV}_{\mathrm{TH}}$ to familiarize the volunteers with the experimental protocol. On the $4^{\text {th }}$ visit, participants performed a constant load exercise at $120 \%$ of $\mathrm{HRV}_{\text {TH }}$ for 30 minutes, in which HR and HRV were constantly recorded. A HR monitor (RS800cx, Polar, Finland) recorded the HR and HRV. Polar Pro Trainer 5 Software (Polar, Finland) was used to download and analyze the $\mathrm{R}-\mathrm{R}$ interval data. The standard deviation one (SD1) of the R-R interval was calculated using Poincaré plots for every minute by Kubios HRV software (Kuopio, Finland). The $\mathrm{HRV}_{\text {TH }}$ was considered as the $1^{\text {st }}$ workload during the maximal incremental exercise test, in which SD1 was lower than 3 milliseconds (Lima and Kiss, 1999).

Basketball players (athletic group)

Basketball players participated in 3 official matches at the same time of day. The 3 assessed official matches were played at the home venue of the assessed team and during the regular competitive season; the entire investigation period lasted 6 weeks. Subjects arrived at the playing venue approximately 90 min before the beginning of the matches. Each investigated match was preceded by a standard 30-min warm-up comprising light aerobic exercise, basketball and team drills, and stretching of the major muscle groups. During the basketball matches, participants were encouraged to drink water during the quarterly breaks to maintain their hydration status. No weight management strategies were implemented and no difference in body mass from pre- to post-match was observed for players for either assessed match. As there was no significant difference between matches for both pre-and post-saliva values, the pooled data of the 3 occasions were retained for further analysis.

\section{Saliva collection and assessments}

The saliva sample was obtained by passive drool, in a sterile $15-\mathrm{ml}$ centrifuge tube 
over a 5-minute period, at rest, before the beginning of the exercise (pre) for the sedentary group, and before the warm-up procedures of each match for the basketball players. The postsampling was conducted approximately $15 \mathrm{~min}$ to 20 min following exercise or a match. No food was taken $90 \mathrm{~min}$ before the first saliva sampling. The 3 matches were played at the same time of day (between 19:00 and 21:00 h) and the reference team won the 3 matches. The sedentary group began the testing procedures at the same time of the day as that in which the basketball players participated in the official matches. After collection, samples were stored at $-80^{\circ} \mathrm{C}$ until the assay. After thawing and centrifugation $(1500 \mathrm{~g}$ for $15 \mathrm{~min}$ ), samples were tested for salivary cortisol concentration and BDNF concentration using enzyme-linked immunosorbent assays (ELISA, Salimetrics ${ }^{\mathrm{TM}}$ and Abnova ${ }^{\mathrm{TM}}$ expanded range kit, respectively) according to the manufacturers' recommendations. The BDNF saliva assay was performed according to manufacturer recommendation procedures for serum and plasma analyses, but as adopted by Virjen et al. (2017), saliva samples were not diluted. The sensitivity provided by the manufacturer is $<15$ $\mathrm{pg} / \mathrm{ml}$, with a detection range of $32.2-2000 \mathrm{pg} / \mathrm{ml}$. All the analyzed samples felt into this range. Salivary cortisol analyses were conducted in accordance with previously adopted procedures (Moreira et al., 2012a, 2012b), and the minimum detection limit was $0.33 \mathrm{nmol} / \mathrm{L}$. The samples were analysed in duplicate and the average intraassay coefficients of variation for the cortisol and BDNF assays were less than $3.6 \%$ and $8 \%$, respectively.

\section{Statistical analysis}

A mixed model 2-way analysis of variance for repeated measures was used to examine differences in salivary cortisol and BDNF between groups and time-points (pre- and postexercise) after checking for data normality (Shapiro-Wilk's test) and homoscedasticity (Levene's test). The sphericity of data was assumed according to Mauchly's test results. In the event of a significant difference, a Bonferroni post-hoc test was used to identify any localized effects. The relationship between the change in salivary cortisol and BDNF from pre to post exercise (and match) was evaluated using Pearson's product moment correlation. The correlation magnitudes were considered as trivial $(\mathrm{r}<0.1)$, small $(\mathrm{r}>0.1-0.3)$, moderate $(\mathrm{r}>0.3-0.5)$, large $(r>0.5-0.7)$, very large $(r>0.7-0.9)$, nearly perfect $(r>0.9)$ and perfect $(r=1.0)$ (Hopkins et al., 2009). The software SPSS (v. 20.0, SPSS Inc., Chicago, IL, USA) was used for data analyses. Statistical significance was set at $p \leq 0.05$.

\section{Results}

Figure 1 shows the results for BDNF concentration in the groups. A significant group effect was observed ( $\mathrm{F}=14.2, p<0.001)$, but there was no effect of interaction $(\mathrm{F}=0.003, p>0.05)$. A higher resting salivary BDNF concentration (pre) and a higher salivary BDNF concentration after the exercise (post) were observed in basketball players, compared to sedentary individuals. However, no change in salivary BDNF was observed for either group between pre and post exercise $(F=0.61, p>0.05)$.

Figure 2 presents the results for salivary cortisol in the groups. A significant interaction effect $(\mathrm{F}=16.8 ; p<0.001)$, and a significant effect for group $(\mathrm{F}=8.9 ; p<0.001)$, and time $(\mathrm{F}=17.9, p<$ 0.001 ) was observed. An increase in salivary cortisol concentration from pre to post match was observed for basketball players, while no change for the sedentary group was verified. Additionally, salivary cortisol concentration was higher for basketball players compared to the sedentary group, only at the post-collection time.

The correlations between the change (\%) in salivary cortisol and BDNF are shown in Figure 3, for basketball players (A) $(\mathrm{r}=-0.11$; trivial) and sedentary individuals $(\mathrm{B})(\mathrm{r}=0.13$; trivial). There was no significant relationship $(p>0.05)$ between the change in salivary cortisol and BDNF for either group.

\section{Discussion}

The aims of this study were to: 1 ) compare salivary cortisol and BDNF concentration before and after physical exercise in sedentary individuals and elite male basketball players, and 2) examine the association between the change in salivary cortisol and BDNF. It was hypothesized that basketball players would present a higher resting BDNF concentration compared to sedentary individuals. This hypothesis was suggested based on recent findings of Oztasyonar (2016), who reported a higher basal blood BDNF 
level in athletes compared to sedentary individuals. The author speculated that this result might be due to the higher attention and concentration demands placed on athletes during combat sports. Despite being speculative, this hypothesis might be plausible. The present results corroborate Oztasyonar's findings and add novel and important information to the literature, since salivary BDNF concentration was compared in sedentary individuals and team-sport athletes for the first time. Taking the results provided by Oztasyonar (2016) together with the findings of the present study, it seems that using either blood or saliva, as a means of determining BDNF concentration, a higher BDNF level in athletes (at least for team-sport and combat-sport athletes) would be observed when compared to sedentary individuals.
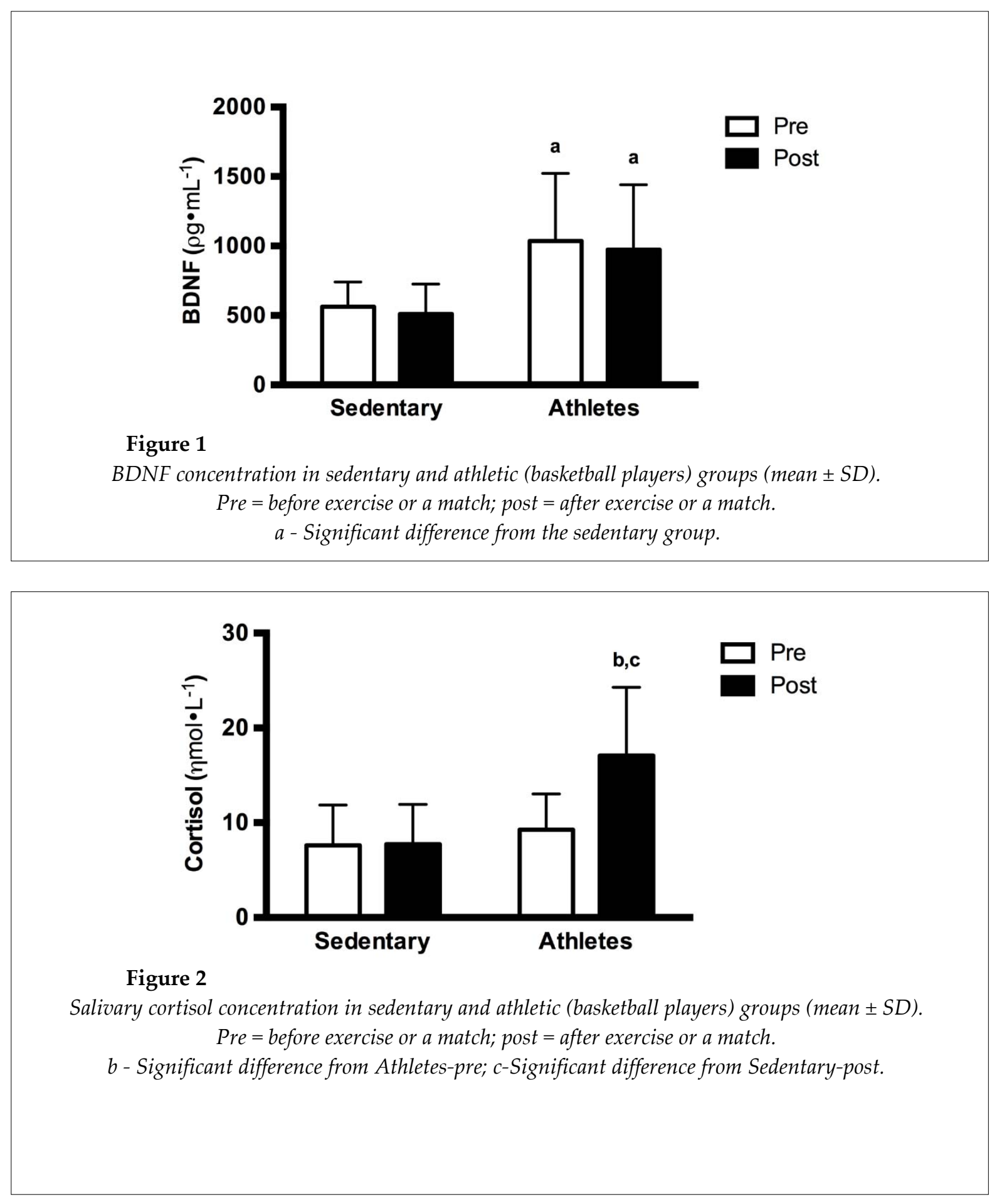

(C) Editorial Committee of Journal of Human Kinetics 


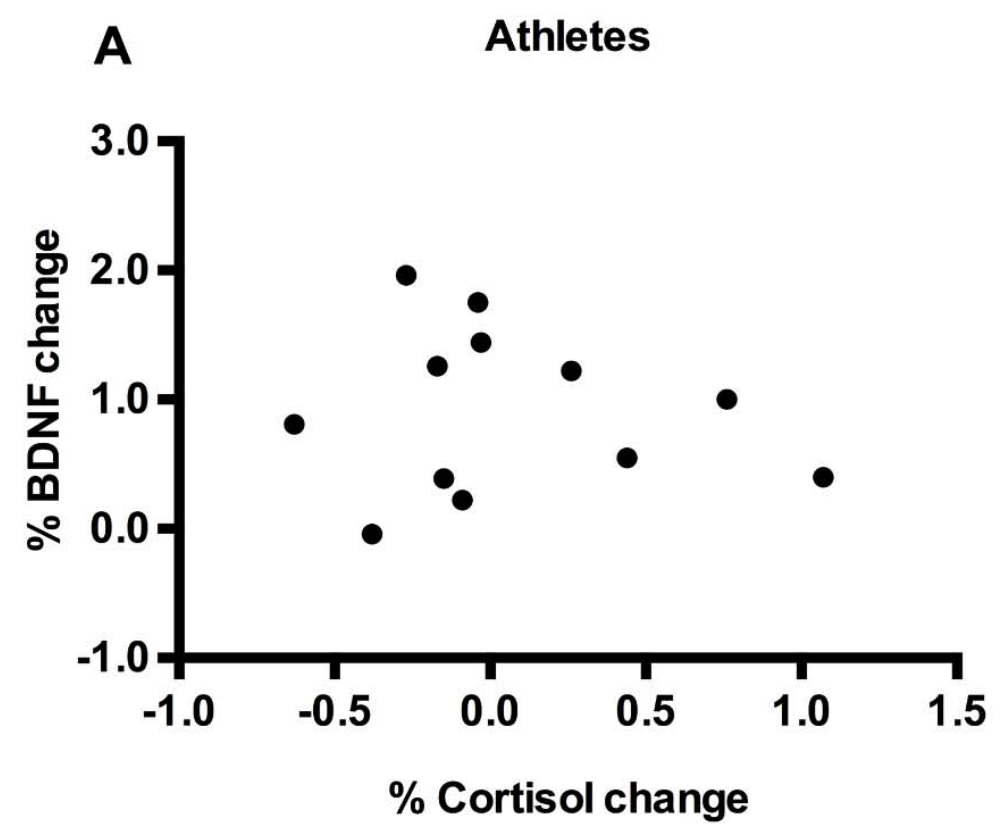

B Sedentary

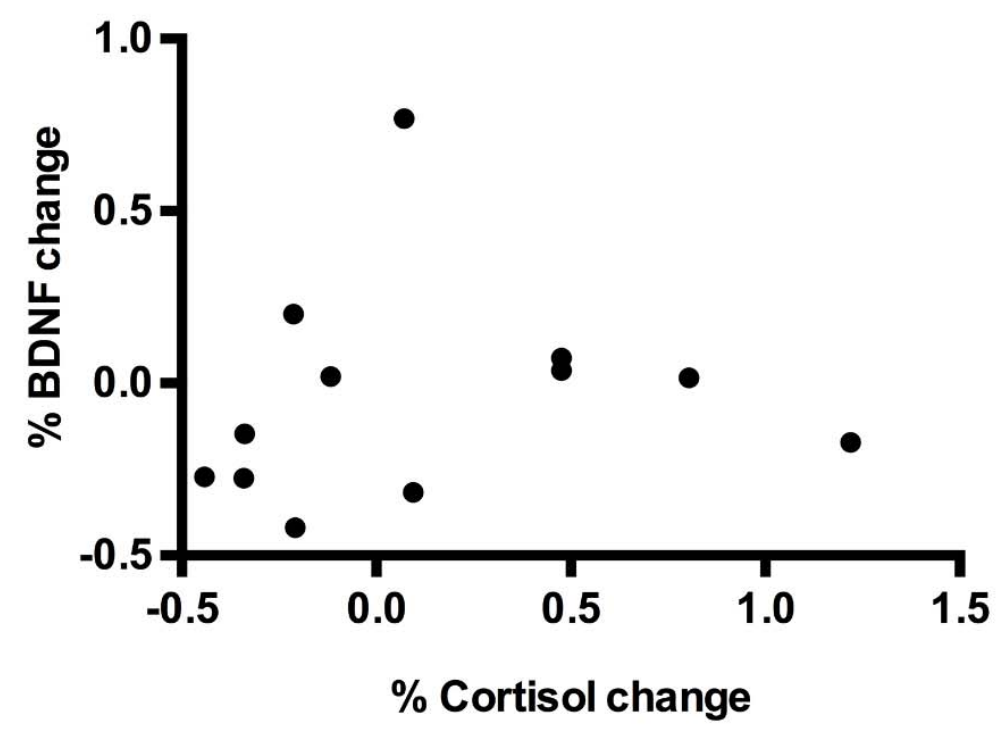

Figure 3

Correlation between changes in salivary BDNF and cortisol for basketball players ( $A$ ) and sedentary individuals $(B)$. 
However, these results are in conflict with those provided by Nofuji et al. (2008) who showed a lower serum BDNF level in trained men compared to sedentary individuals, and reported negative correlations between serum BDNF concentration and daily total energy expenditure $(r=-0.50)$, movement-related energy expenditure $(\mathrm{r}=-0.50)$, and walking count $(\mathrm{r}=-0.48)$. As it is still hard to determine whether some causal relationships exist between regular exercise and peripheral BDNF release, further studies focused on explaining not only these relationships, but also the mechanisms involved are, therefore, warranted. Despite the need for evidence to corroborate or refute the positive role of exercise on BDNF concentration, it seems likely that, in general, one could expect to find a positive association. Data from future studies comparing athletes from different sports and with different training experience (elite athletes versus recreational athletes, male versus female athletes, team-sports versus combat-sports athletes, etc.) may aid in advancing the understanding of the BDNF response in athletes, as well as its mechanisms.

It was expected that BDNF would increase from pre to post exercise and that this change could be associated with the change in salivary cortisol, adopted as a marker of psychophysiological stress. However, no significant change in salivary BDNF was verified for either of the groups, from pre to post exercise, despite a significant increase in salivary cortisol for basketball players. In addition, there was no significant correlation between the change in salivary BDNF and cortisol for both groups.

Although few studies have been conducted in humans using salivary BDNF, the hypothesis that BDNF concentration would increase either after official basketball matches in the elite players, or after exercising at a constant high-intensity load in sedentary individuals, was formulated based on the proposed role of BDNF in protecting the human body from stress and increased arousal (Saruta et al., 2010b; Tsukinoki et al., 2007) and also taking into account the results from Tsukinoki et al. (2006) and Saruta et al. $(2010 a, b)$, who demonstrated a significant increase in plasma BDNF concentration in rats that underwent stress models. These authors attributed these responses to BDNF secretion from the salivary gland into the circulation. A possible explanation for the absence of a significant change in salivary BDNF in the present study might be that the salivary level of BDNF may not reflect the parallel increments that occur locally in the brain and muscle. This speculation was addressed by Forti et al. (2014), but the authors were referring to plasma BDNF, which might be then extended to salivary BDNF, as both may be representative means of evaluating periphery BDNF responses to exercise. Nevertheless, whilst this might be a possible explanation for the lack of change in BDNF in the present study and others (Goekint et al., 2010; Rojas-Vega et al., 2012 ), this hypothesis is still largely speculative and needs further confirmation.

Another issue that deserves discussion is the intensity of the performed exercises. For example, Ferris et al. (2007) showed the role of intensity in eliciting an acute change in the BDNF level as a result of physical exercise. Rojas-Vega et al. (2006) evaluated the effect of short-term aerobic exercise and a ramp-incremental cycle ergometer test to exhaustion on the acute response of serum BDNF, capillary blood lactate (LA) and cortisol in 8 male athletes. The results of their study showed that BDNF, LA, and cortisol did not change during the warm-up period, but that LA was increased $(p<0.05)$ at the end of the ramp test that followed the warm-up period, and during the recovery period. Serum BDNF was only increased at the point of exhaustion $(\mathrm{p}<$ $0.05)$, while no significant difference was found for resting concentration and the concentration during the recovery period. Moreover, the cortisol concentration increased at 10 and $15 \mathrm{~min}$ post incremental exercise, compared to rest.

While these results suggest that the intensity of the exercise might be a key factor in inducing BDNF change, the results of the present study, however, highlighted that other factors and variables rather than training intensity, mode and the physical fitness of the subjects might play a role in acute BDNF responses. The present findings add still more complexity to the understanding of BDNF responses to exercise, but they also contribute to the literature as they describe, for the first time, the lack of change in acute salivary BDNF while performing highintensity exercise, regardless of the physical fitness of the assessed subjects (sedentary 
individuals vs. elite athletes) or the mode of the exercise (official basketball matches; intermittent exercise vs. high-intensity constant exercise).

Despite performing exercise in the severe intensity domain, salivary cortisol concentration did not change from pre to post exercise in the sedentary group, while a significant increase was observed in athletes. This result suggests that the inherent psychological stress associated to official matches plays a key role in mediating the HPA axis activity in response to stress and increased arousal activity. However, the absence of changes in salivary cortisol in the sedentary group was partially unexpected. Exercising in the severe intensity domain was expected to induce an increase in cortisol from pre to post exercise in the sedentary subjects due to the permissive effects of cortisol, which allow, for example, catecholamines and other sympathetic products to exert effects on the cardiovascular system, and the critical role of cortisol in metabolism by mobilizing energy resources (Viru and Viru, 2004). It is possible that the duration of the exercise $(30 \mathrm{~min})$ may explain at least in part why a change in cortisol concentration was not observed. Additionally, it might be speculated that this lack of salivary cortisol changes would be associated with the mild psychological stress experienced by the subjects during the exercise task (Dickerson and Kemeny, 2004).

In contrast to the exercise performed by the sedentary subjects, basketball players were involved in a typical stress situation, which may be characterized by social-evaluative threat and an uncontrollable condition, as the results and output of the match are largely unpredictable and uncertain. The increase in salivary cortisol observed in the players evaluated in the present study, is in line with several previous findings that have demonstrated that the additional level of stress associated with real competition settings leads to a greater increase in salivary cortisol concentration in basketball players (Moreira et al., 2012a, 2012b), weightlifting athletes (Crewther et al., 2011) and Jiu-Jitsu athletes (Moreira et al., 2012c). Again, it is worth noting that, despite this significant increase in salivary cortisol concentration for basketball players, no significant change in BDNF was found. This finding suggests that different pathways might be involved in salivary cortisol and salivary BDNF responses, and also that the change in salivary cortisol may not influence the salivary BDNF response.

Among the limitations of the present study, the use of commercial kits that have not yet been specifically validated for saliva analyses should be highlighted (Vrijen et al., 2017). However, it is worth mentioning that the BDNF kit used in the present study allowed the detection of the presence of BDNF in saliva. Caution is also needed to further compare the results of the present study with others using different commercial kits or adopting different collection, storage and analysing methods. The fact that data obtained from a single basketball team was used in the present study also needs to be considered, as differences in training routine, physical and technical level, and competition environment might affect results.

In summary, the present findings show a higher salivary BDNF concentration in athletes, when compared to sedentary individuals. Increased salivary cortisol from pre to post physical exertion was observed for basketball players, but not for the sedentary group. Additionally, no changes in salivary BDNF, for either the sedentary or athlete groups, were observed from pre to post exercise. These results might suggest that the higher salivary BDNF concentration observed for athletes might be associated with the repeated exposure of the players to stressful competition situations that ultimately lead to a higher salivary BDNF level. Highly speculatively, we suggest that this result might occur due to the proposed association between BDNF concentration and its role in protecting from stress and increased arousal, as well as the constant and elevated level of attention and concentration associated with decisionmaking, which occurs during basketball matches. This result suggests that even when a highintensity exercise is performed, the change in salivary BDNF might not be an expected result, regardless of the physical fitness level of the assessed subjects (sedentary individuals vs. elite athletes) or the mode of the exercise (official basketball matches; intermittent exercise vs. highintensity constant exercise). Finally, the present results also suggest that different mechanisms might be involved in salivary cortisol and salivary BDNF responses during physical exertion. 


\section{Acknowledgements}

We would like to thank all participants, basketball players, and research support staff involved in this study for their committed participation.

This research was supported by the Brazilian National Council for Scientific and Technological Development (Conselho Nacional de Desenvolvimento Científico e Tecnológico; CNPq) of the Minister of Science and Technology (Ministério da Ciência e Tecnologia, Inovações e Comunicações; MCTIC) (Call - MCT/CNPq № 14/2011 - Universal), and by the Fundação de Amparo à Pesquisa do Estado de São Paulo (São Paulo Research Foundation; Grant 2008/10404-3) awarded to the first author. This study was financed in part by the "Coordenação de Aperfeiçoamento de Pessoal de Nível Superior". Brasil (CAPES). Finance Code 001 .

\section{References}

Aardal E, Holm AC. Cortisol in saliva-reference ranges and relation to cortisol in serum. Eur J Clin Chem Clin Biochem, 1995; 33: 927-932

Crewther BT, Heke T, Keogh JW. The effects of training volume and competition on the salivary cortisol concentrations of Olympic weightlifters. J Strength Cond Res, 2011; 25: 10-15

Dickerson SS, Kemeny ME. Acute stressors and cortisol responses: A theoretical integration and synthesis of laboratory research. Psychol Bull, 2004; 130: 355-391. doi: 10.1037/0033-2909.130.3.355

Ferris LT, Williams JS, Shen CL. The effect of acute exercise on serum brain-derived neurotrophic factor levels and cognitive function. Med Sci Sports Exerc, 2007; 39: 728-734. doi: 10.1249/mss.0b013e31802f04c7

Forti LN, Njemini R, Beyer I, Eelbode E, Meeusen R, Mets T, Bautmans, I. Strength training reduces circulating interleukin-6 but not brain-derived neurotrophic factor in community-dwelling elderly individuals. Age (Dordr), 2014; 36: 9704. doi: 10.1007/s11357-014-9704-6

Goekint M, Roelands B, De Pauw K, Knaepen K, Bos I, Meeusen R. Does a period of detraining cause a decrease in serum brain-derived neurotrophic factor? Neurosci Lett, 2010; 486: 146-149. doi:10.1016/j.neulet.2010.09.032

Gozansky WS, Lynn JS, Laudenslager ML, Kohrt WM. Salivary cortisol determined by enzyme immunoassay is preferable to serum total cortisol for assessment of dynamic hypothalamic-pituitaryadrenal axis activity. Clin Endocrinol (Oxf), 2005; 63: 336-341

Hofer M, Pagliusi SR, Hohn A, Leibrock J, Barde YA. Regional distribution of brain-derived neurotrophic factor mRNA in the adult mouse brain. EMBO J, 1990; 9: 2459-2464

Hopkins WG, Marshall SW, Batterham AM, Hanin J. Progressive statistics for studies in sports medicine and exercise science. Med Sci Sports Exerc, 2009; 41: 3-13. doi 10.1249/MSS.0b013e31818cb278

Lewin G, Barde Y. Physiology of the neurotrophins. Annu Rev Neurosci, 1996; 19: 289-317

Lima JRP, Kiss MAPD. Heart rate variability threshold. Rev Bras Ativ Fís Saúde, 1999; 4: 29-38

Mandel AL, Ozdener $\mathrm{H}$, Utermohlen V. Identification of pro- and mature brainderived neurotrophic factor in human saliva. Arch Oral Biol, 2009; 54: 689-695. doi: 10.1016/j.archoralbio.2009.04.005

Matsuki C, To M, Kondo Y, Sugiyama H, Yamamoto Y, Shimizu T, Kamata Y, Saruta J, Tsukinoki K. Associations between brain-derived neurotrophic factor and estradiol in women's saliva. Neuro Endocrinol Lett, 2014; 35: 236-241

Moreira A, Crewther BT, Freitas CG, Arruda AFS, Costa EC, Aoki MS. Session RPE and salivary immuneendocrine responses to simulated and official basketball matches in elite young male athletes. J Sports Med Phys Fitness, 2012a; 52: 682-687 
Moreira A, McGuigan M, Arruda AFS, Freitas CG, Aoki MS. Monitoring internal lad parameters during simulated and official basketball matches. J Strength Cond Res, 2012b; 26: 861-866. doi: 10.1519/JSC.0b013e31822645e9

Moreira A, Franchini E, Freitas CG, Arruda AFS, Moura NR, Costa EC, Aoki MS. Salivary cortisol and immunoglobulin A responses to simulated and official Jiu-Jitsu matches. J Strength Cond Res, 2012c; 26: 2185-2191

Nofuji Y, Suwa M, Moriyama Y, Nakano H, Ichimiya A, Nishichi R, Sasaki H, Radak Z, Kumagai S. Decreased serum brain-derived neurotrophic factor in trained men. Neurosci Lett, 2008; 437: 29-32. doi: 10.1016/j.neulet.2008.03.057

Oztasyonar Y. Interaction between different sports branches such as taekwondo, box, athletes and serum brain derived neurotrophic factor (BDNF) levels. J Sports Med Phys Fitness, 2016; 57: 457-460. doi: 10.23736/S0022-4707.16.06070-X

Papacosta E, Nassis GP. Saliva as a tool for monitoring steroid, peptide and immune markers in sport and exercise science. J Sci Med Sport, 2011; 14: 424-434. doi: 10.1016/j.jsams.2011.03.004

Rojas-Vega S, Hollmann W, Vera Wahrmann B, Strüder HK. pH Buffering does not influence BDNF responses to Exercise. Int J Sports Med, 2012; 33: 8-12. doi: 10.1055/s-0031-1285929

Rojas-Vega S, Strüder HK, Vera Wahrmann B, Schmidt A, Bloch W, Hollmann W. Acute BDNF and cortisol response to low intensity exercise and following ramp incremental exercise to exhaustion in humans. Brain Res, 2006; 1121: 59-65. doi: 10.1016/j.brainres.2006.08.105

Russo-Neustadt A, Ha T, Ramirez R, Kesslak JP. Physical activity-antidepressant treatment combination: Impact on brain-derived neurotrophic factor and behavior in an animal model. Behav Brain Res, 2001; 120: 87-95. doi: 10.1016/S0166-4328(00)00364-8

Sapolsky RM, Romero LM, Munck AU. How do glucocorticoids influence stress responses? Integrating permissive, suppressive, stimulatory, and preparative actions. Endocr Rev, 2000; 21: 55-89. doi: $\underline{10.1210 / \text { edrv.21.1.0389 }}$

Saruta J, Fujino K, To M, Tsukinoki K. Expression and localization of brain-derived neurotrophic factor (BDNF) mRNA and protein in human submandibular gland. Acta Histochem Cytochem, 2012; 45: 211218. http://dx.doi.org/10.1267/ahc.12005

Saruta J, Kondo Y, Sato C, Shiiki N, Tsukinoki K, Sato S. Salivary glands as the source of plasma brainderived neurotrophic factor in stressed rats engaged in biting behavior. Stress, 2010a; 13: 238-247. doi: $10.3109 / 10253890903296728$

Saruta J, Lee T, Shirasu M, Takahashi T, Sato C, Sato S, Tsukinoki K. Chronic stress affects the expression of brain-derived neurotrophic factor in rat salivary glands. Stress, 2010b; 13: 53-60. doi: $10.3109 / 10253890902875167$

Tang SW, Chu E, Hui T, Helmeste D, Law C. Influence of exercise on serum brain-derived neurotrophic factor concentrations in healthy human subjects. Neurosci Lett, 2008; 431: 62-65. doi: 10.1016/j.neulet.2007.11.019

Tirassa P, Iannitelli A, Sornelli F, Cirulli F, Mazza M, Calza A, Alleva E, Branchi I, Aloe L, Bersani G, Pacitti F. Daily serum and salivary BDNF levels correlate with morning-evening personality type in women and are affected by light therapy. Riv Psichiatr, 2012; 47: 527-534. doi: 10.1708/1183.13096

Törnhage CJ. Salivary cortisol for assessment of Hypothalamic-Pituitary-Adrenal axis function. Neuroimmunomodulation, 2009; 16: 284-289

Tsukinoki K, Saruta J, Muto N, Sasaguri K, Sato S, Tan-Ishii N, Watanabe Y. Submandibular Glands Contribute to Increases in Plasma BDNF Levels. J Dent Res, 2007; 86: 260-264. doi: $10.1177 / 154405910708600312$

Tsukinoki K, Saruta J, Sasaguri K, Miyoshi Y, Jinbu Y, Kusama M, Sato S, Watanabe Y. Immobilization Stress Induces BDNF in Rat Submandibular Glands. J Dent Res, 2006; 85: 844-848. doi: $10.1177 / 154405910608500913$ 
Vaynman SS, Ying Z, Yin D, Gomez-Pinilla F. Exercise differentially regulates synaptic proteins associated to the function of BDNF. Brain Res, 2006; 1070: 124-130. doi: 10.1016/j.brainres.2005.11.062

Viru A, Viru M. Cortisol - Essential adaptation hormone in exercise. Int J Sports Med, 2004; 25: 461-464. doi: 10.1055/s-2004-821068

Vrijen C, Schenk HM, Hartman CA, Oldehinkel AJ. Measuring BDNF in saliva using commercial ELISA: Results from a small pilot study. Psychiatry Res, 2017; 254: 340-346. doi: 10.1016/j.psychres.2017.04.034

World Health Organization. Quality of Life assessment (WHOQOL): position paper from the World Health Organization. Soc Sci Med, 1995; 41: 1403-1409

\section{Corresponding author:}

\section{Dr. Alexandre Moreira}

School of Physical Education and Sport, University of Sao Paulo, Sao Paulo, Brazil.

Av. Prof. Mello Moraes, 65, Cidade Universitária, São Paulo, Brazil. Zip Code 05508-030

E-mail: alemoreira@usp.br 